\title{
COMPOSIÇÃO E DISTRIBUIÇÃO dO FITOPLÂNCTON EM ÁREAS COSTEIRAS E OCEÂNICAS DA REGIÃO SUESTE DO BRASIL
}

\author{
Frederico Pereira Brandini * \\ Cristine Louise Braum Moraes
}

\section{ABSTRACT}

Total phytoplankton samples and hydrographical parameters were collected from the surface of 16 stations located in the southeastern sector of the brazilian coast during the autumn cruise of the R/V "Almirante Saldanha". The limits of tem. perature $\left(22-25^{\circ} \mathrm{C}\right)$ and salinity $\left(35,0-37,1 \times 10^{-3}\right)$ observed indicated the great influence of the Tropical Water over the sampling area where the oligotrophic condition is certainly due to the low nutrient concentrations of this watermass. Phosphate, nitrate and silicate ranged from 0.0 to $0.63,1.41$ to 5.69 and from 4.10 to $25,25 \mu \mathrm{g}$-at. $\mathrm{l}^{-1}$, respectively.

The most frequent diatoms were Coscinodiscus spp, Coscinosira $\mathrm{sp}$, Pleurosigma $\mathrm{sp}$, Pseudoeunotia doliolus, Thalassionema nitzschioides, Thalassiothrix frauenfeldii and $T$. mediterranea, among 49 species observed. Prorocentrum and Protoperidinium spp dominated the dinoflagellate population although the genus Ceratium was represented by a greater number of species. Cyanophycean cells were abundant in coastal (Anabaena sp) and oceanic (Trichodesmium erythraeum) samples.

The phytoplankton community was numerically dominated by nanoplanktonic organisms like phytoflagellates (including mo nads) and the coccolithophorid Coccolithus huxleyi. The phytoplankton association observed in the present study is typical of oligotrophic warm waters of the Brazil Current.

KEY WORDS: Phytoplankton, Composition, Distribution, Southeastern Coast (Brazil).

\footnotetext{
- Centro de Biologia Marinha - UFPR

Av. Beira Mar $s / n$ - Pontal do Sul

Paranaguá - Paraná - 83.200
} 


\section{RESUMO}

Amostras de fitoplâncton total e parâmetros hidrográficos foram obtidos na superfície de 16 estações localizadas no setor sudeste da costa brasileira durante o outono de 1983. Os limites de temperatura $\left(22-25^{\circ} \mathrm{C}\right)$ e salinidade $(35,0-37,1 \mathrm{x}$ $10^{-3}$ ) observados indicaram a influência da Água Tropical na área estudada onde as condições oligotróficas são devidas à baixa concentração de nutrientes nessa massa d'água. Fosfato, nitrato e silicato variaram entre 0,0 e $0,63,1,41$ e 5,69 , e 4,10 e 25,25 $\mu$ g-at. ${ }^{-1}$, respectivamente.

As diatomáceas mais freqüentes foram Coscinodiscus spp, Coscinosira sp, Pleurosigma sp, Pseudoeunotia doliolus, Thalassionema nitszchioides, Thalassiothrix frauenfeldii e T. mediterranea, dentre as 49 espécies observadas. Prorocentrum e Protoperidinium spp dominaram a população de dinoflagelados apesar do gênero Ceratium ter apresentado o maior número de espécies. As cianofíceas Anabaena sp e Oscillatoria (=Trichodesmium) erythraeum foram abundantes nas amostras costeiras e oceânicas.

A comuindade fitoplanctônica foi numericamente dominada por organismos do nanoplâncton, tais como fitoflagelados (incluindo-se mônadas) e o cocolitoforídeo Coccolithus huxleyi. A associação fitoplanctônica observada no presente trabalho é típica das águas quentes e oligotróficas da Corrente do Brasil.

PALAVRAS CHAVE: Fitoplâncton, composição, distribuição

Região Sueste (Brasil)

\section{INTRODUÇÃO}

A maioria dos trabalhos recentes sobre a composição e a biomassa do fitoplâncton da região sueste do Brasil concentram-se nas áreas costeiras (BRANDINI, 1982, 1985; KUTNER, 1974; MOREIRA et al., 1975; MOREIRA \& MOREIRA, 1978; etc.) Em regiões de plataforma afastadas da costa e em áreas oceânicas, são poucos os estudos realizados até o presente (SOA RES, 1983; BRANDINI, 1986). Recentemente, BRANDINI (1986) observou a distribuição geográfica dos principais grupos fitoplanctônicos em relação ao padrão de distribuição de massas d'água durante 0 inverno. $O$ presente trabalho tem por obje- 
tivo descrever a composição e a distribuição do fitoplâncton de águas costeiras e oceânicas da região sueste brasileira durante o outono de 1982 e suas relações com as condições hidrográficas. Os períodos de verão e primavera serão objeto de futuros estudos.

\section{MATERIAL E MÉTODOS}

O material estudado foi obtido no decorrer da OPERAÇÃO SUESTE II (DHN, 1983), à bordo do N/Oc. Almirante Saldanha, em 16 estações oceanográficas distribuidas ao longo da região costeira e oceânica entre São Paulo e Santa Catarina (Fig. 1). Por problemas logísticos não foi possível a coleta em um número maior de estações.

Os dados físico-químicos foram fornecidos pelo Banco Nacional de Dados Oceanográficos (DHN - Rio de Janeiro). A temperatura e a salinidade foram medidas utilizando-se respectivamente o termômetro de reversão acoplado à garrafa de Nansen e um salinômetro de indução GRUNDY (mod. 623ON). As concentrações de nitrato, fosfato e silicato foram medidas a bordo utilizando-se as técnicas descritas por STRICKLAND \& PARSONS (1972).

As amostras de água para o estudo do fitoplâncton total foram coletadas com balde plástico e fixadas com formol neutralizado até a concentração final de $0,4 \%$.

Posteriormente, $1000 \mathrm{ml}$ foram sedimentados durante uma semana e concentrados para $100 \mathrm{ml}$ no laboratório do Centro de Biologia Marinha da Universidade Federal do Paraná. As amostras concentradas foram coradas com Rosa de Bengala e analisadas pela técnica de UTERMÖHL (1958), utilizando-se cubas de sedimentação de $25 \mathrm{ml}$.

As células maiores do que $20 \mu \mathrm{m}$ foram contadas com aumento de $160 \mathrm{X}$ e os grupos nanoplanctônicos $(<20 \mu \mathrm{m})$ foram contados com aumento de $400 \mathrm{X}$ em um número suficiente de transectos até que a quantidade de organismos dominantes fosse superior a 100 . De acordo com esse procedimento o êrro de contagem é da ordem de $24 \%$ (VENRICK, 1978; EDLER, 1979). 
A análise da clorofila a foi feita pelo método tricromático após a extração dos pigmentos com acetona $90 \%$ de acordo com as recomendações e as equações do SCOR-UNESCO (1963).

\section{RESULTADOS}

A Tabela I apresenta os resultados físico-químicos e de clorofila a obtidos na camada superficial das estações amostradas. A- temperatura e a salinidade a 10 metros variaram respectivamente de 22,0 a $24,7^{\circ} \mathrm{C}$ e de 35,0 a $37,1 \times 10^{-3}$ e, de um modo geral, os máximos foram observados nas áreas oceânicas. Valores mínimos de temperatura entre 22 e $23^{\circ} \mathrm{C}$ e salinidades entre 35,0 e $35,5 \times 10^{-3}$ foram registrados nas estações costei ras 6213,6214 e 6240 , e na estação 6271 localizada sobre a plataforma mais extensa do Estado de São Paulo. A salinidade mínima de $33,05 \times 10^{-3}$ foi obtida em frente a Santos. Na radial da Ilha de Santa Catarina (est. 6213 - 6218), a temperatura e a salinidade aumentaram gradativamente em direção à região oceânica.

As concentrações de nitrato, fosfato e silicato na superfície variaram respectivamente de 1,41 a $5,69,0,0$ a 0,63 e de 4,10 a $25,25 \mu \mathrm{g}$-at. $\mathrm{I}^{-1}$. As concentrações de fosfato e silicato foram bastante elevadas ao longo da radial da Ilha de Santa Catarina Nas demais estações localizadas sobre a plataforma ao norte da área estudada, as concentrações de fosfato e silicato foram mais baixas com valores inferiores a $0,2 \mu$ g-at. $I^{-1}$ e entre 5 e $8 \mu \mathrm{g}$-at. $\mathrm{I}^{-1}$, respectivamente. As concentrações de nitrato foram em geral baixas e em torno de $2 \mu \mathrm{g}$-at. $\mathrm{l}^{-1}$, com exceção da estação 6240 onde foi medida a concentração máxima de 5,7 $\mu$ g-at.l. ${ }^{-1}$.

A concentração de clorofila a variou de 0,16 a $1,10 \mu \mathrm{g} .1^{-1}$ sendo que os máximos foram obs̄ervados nas estações costeiras 6213, próximo à llha de Santa Catarina, e 6290 localizadas em frente a Baia de Santos. Valores mínimos em torno de $0,2 \mu \mathrm{g} . l^{-1}$ foram registrados nas áreas oceânicas.

As espécies observadas durante a análise do fitoplâncton total estão listadas na Tabela II. Foram identificadas 49 espécies de diatomáceas, 33 espécies de dinoflagelados, 1 espécie de cocolitoforídeo, 2 espécies de silicoflagelados e 2 espécies 
Tabela I. Parâmetros físico-químicos e concentração de clorofila a na superfície das estações amostradas.

\begin{tabular}{|c|c|c|c|c|c|c|}
\hline Estação & ${ }_{\text {(à }}^{\mathrm{T}}{ }^{\circ} \mathrm{C}$ & $10 \mathrm{~m})^{S \% o}$ & $\mathrm{PO}_{4}-\mathrm{P}$ & $\begin{array}{c}\mathrm{NO}_{3}-\mathrm{N} \\
\left(\mu \mathrm{g}-\mathrm{at} . \mathrm{I}^{-1}\right)\end{array}$ & $\mathrm{SiO}_{2} \mathrm{Si}$ & $\begin{array}{l}\text { Clor. a } \\
\left(\mu \mathrm{g} . \mathrm{I}^{-1}\right)\end{array}$ \\
\hline 6213 & 22,02 & 35,03 & 0,63 & 1,76 & 12,83 & 1,10 \\
\hline 6214 & 22,12 & 35,43 & 0,63 & 1,76 & 14,95 & 0,48 \\
\hline 6215 & 23,14 & 35,62 & 0,39 & 1,82 & 24,85 & - \\
\hline 6216 & 24,55 & 36,81 & 0,47 & 2,91 & 25,25 & - \\
\hline 6217 & 24,18 & 36,47 & 0,36 & 1,42 & 24,64 & 0,16 \\
\hline 6218 & 24,35 & 36,88 & 0,41 & 1,56 & 20,91 & 0,18 \\
\hline 6231 & 24,45 & 36,65 & 0,40 & 1,49 & 16,07 & 0,26 \\
\hline 6240 & 22,45 & 35,21 & 0,23 & 5,69 & 21,32 & 0,82 \\
\hline 6262 & 23,01 & 35,39 & 0,15 & 2,32 & 8,19 & 0,21 \\
\hline 6263 & 23,41 & 36,21 & 0,06 & 2,42 & 4,52 & 0,27 \\
\hline 6266 & 24,74 & 36,91 & 0,0 & 1,97 & 4,10 & 0,21 \\
\hline 6271 & 22,20 & 35,01 & 0,15 & 2,77 & 7,04 & 0,26 \\
\hline 6278 & 22,32 & 35,36 & 0,08 & 1,71 & 6,20 & 0,29 \\
\hline 6282 & 24,55 & 37,12 & 0,0 & 1,48 & 5,15 & 0,16 \\
\hline 6283 & 24,44 & 37,04 & 0,03 & 1,73 & 6,20 & 0,18 \\
\hline 6290 & 22,26 & 33,05 & 0,06 & 1,49 & 9,98 & 0,96 \\
\hline
\end{tabular}


Tabela II. Lista das espécies de fitoplâncton observadas na região Sueste em maio/junho de 1983.

\section{DIATOMACEAS}

Actinoptychus flabellatus

Anphiprora sp

Asteromphalus flabellatus

Bacteriastrum hyalinum

Bacteriastrum sp

Biddulphia mobiliensis

Biddulphia sinensis

Biddulphia sp

Chaetoceros brevis

Chaetoceros laevis

Chaetoceros sp

Cyclolella sp

Climacodium frauenfeldianum

Corethron hystrix

Coscinodiscus nitidus

Coscinodiscus sp

Coscinosira $\mathrm{sp}$

Dactyliosolen cf antarcticus

Dactyliosolen mediterraneus

Diploneis sp

Ditylum brightwellii

Hemiaulus hauckii

Hemiaulus membranaceus

Hemiaulus sinensis

Hemiaulus $s p$

Leptocylindrus danicus

Melosira sulcata

Navicula $\mathrm{sp}$

Nitzchia of seriata

Planktoniella sol

Pleurosigma normanii

Pleurosigma $\mathrm{sp}$

Pseudoeunotia doliolus

Rhizosolenia alata

Rhizosolenia robusta

Rhizosolenia setigera

Rhizosolenia styliformis

Rhizosolenia sp

Skeletonema costatum

Stauroneis $\mathrm{sp}$

Stephanopyxis turris

Streptotheca thamensis

Thalassionema nitzchioide

Thalassiosira subtilis

Thalassiosira $\mathrm{sp}$

Thalassiothrix frauenfeldii

Thalassiothrix longissima

Thalassiothrix mediterransa

Thalassiothrix $\mathrm{sp}$
DINOFLAGELADOS

Anphidinium sp

Ceratium furca

Ceratium fusus

Ceratium gibberum

Ceratium hexacanthum

Ceratium lineatum

Ceratium massiliense

Ceratium cf pentagonum

Ceratium setaceum

Ceratium symetricum

Ceratium trichoceros

Ceratium tripos

Ceratium sp

Cystodinium sp

Dinophysis $\mathrm{sp}$

Gymnodinium sp

Gyrodinium $s p$

Ornithocercus of heteropomus

Ornithocercus sp

Oxytoxum longipes

Oxytoxum scolopax

Oxytoxum sp

Protoperidinium pentagonum

Protoperidinium $\mathrm{sp}$

Podolampas palmipes

Podolampas $\mathrm{sp}$

Prorocentrum gracili

Prorocentrum micans

Prorocentrum obtusidens

Prorccentrum sp

Pyrocystis fusiformis

Pyrocystis lunula

Pyrocystis sp

\section{COCOLITOFORIDEOS}

Coccolithus huxleyi

SILICOFLAGELADOS

Dictyocha fibula

Mesocena sp

\section{CIANOFICEAS}

Anabaena sp

Oscillatoria (Trichodesmium) $\mathrm{sp}$ 
de cianofíceas. As diatomáceas mais freqüentes foram Coscinodiscus spp, Coscinosira sp, Pleurosigma sp, Pseudoeunotia doliolus, Thalassionema nitzschiodes, Thalassiothrix frauenfeldii e T. mediterranea. Skeletonema costatum foi abundante apenas na estação 6215 , próximo ao talude continental.

Os dinoflagelados mais abundantes foram Prorocentrum e Protoperidinium spp. No entanto, o gênero Ceratium apresen tou o maior número de espécies considerando-se toda a área amostrada.

Cocolitoforídeos e silicoflagelados foram dominados por Coccolithus huxleyi e Dictyocha fibula, respectivamente. Dentre as cianofíceas, Anabaena sp dominou as associações costeiras e na estação oceânica 6266. Oscillatoria (=Trichodesmium) erythraeum foi mais abundante nas áreas mais afastadas da costa.

A Fig. 2 indica a distribuição e a composição geral do fitoplâncton total na área estudada. Os fitoflagelados (incluindo-se mônadas) e Coccolithus huxleyi foram numericamente do. minantes na maioria das amostras com máximos de $827 \times 10^{3}$ céls/l (est. 6214) e $584 \times 10^{3}$ (est. 6240), respectivamente. Por problemas técnicos, os fitoflagelados e mônadas só foram enumerados nas estações ao longo da radial de Santa Catarina e nas estações 6240, 6262, 6263 e 6266. Na radial de Santa Catarina, o nanoplancton foi abundante tanto em áreas costejras quanto oceânicas sendo que os fitoflagelados e cocolitoforídeos dominaram igualmente nas áreas sobre a plataforma e nas regiões oceânicas; diatomáceas e dinoflagelados decresceram em direção às áreas oceânicas e os máximos de $1,1 \times 10$ e $3,7 \times 10^{3}$ céls/l, respectivamente, foram observados na estação 6213. Ao norte da área estudada a distribuição de diatomáceas e dinoflagelados não apresentou um padrão definido e o mesmo foi observado com relação aos fitoflagelados e cocoli toforídeos. Os silicoflagelados foram pouco abundantes e ocorreram apenas nas estações costeiras do litoral catarinense.

\section{DISCUSSÃO}

A região estudada, compreendida entre 23 e $30^{\circ} \mathrm{S}$ e entre 45 e $49^{\circ} \mathrm{W}$, está localizada imediatamente ao norte da Convergência Subtropical onde ocorre o encontro de águas tropicais 
oligotróficas com águas frias de origem subantártica mais ricas em nutrientes. Esse encontro de massas d'água afeta intensamente as condições hidrográficas da região sul do Brasil nos meses de inverno (EMILSSON, 1961; MIRANDA et al., 1973) e, conseqüentemente, altera completamente os padrões de distribuição geográfica da população fitoplanctônica (BRANDINI, 1986). No presente trabalho, os pares termohalinos obtidos nas camadas superficiais caracterizam a massa de Água Tropical e a massa de Água de Plataforma de acordo com EMILSSON (1961).

As maiores concentrações de fosfato e silicato observadas nas estações do litoral catarinense são provavelmente devidas à drenagem continental do Rio de La Plata e da Lagoa dos Patos trazida por correntes costeiras em direção ao norte (PALACIO, 1982). É interessante observar que as concentrações de nitrato e silicato na radial da llha de Santa Catarina aumentaram da costa em direção às áreas oceânicas até a estação 6216 localizada próximo à linha do talude continental. Isto pode estar associado à ressurgência de borda de plataforma já mencionada por alguns autores como sendo um fenômeno freqüente na região sueste (MESQUITA et al., 1983; BRANDINI, 1986). No entanto, as baixas concentrações de nitrato observadas em todas as amostras caracterizam, de um modo geral, as condições oligotróficas da Água Tropical transportada pela Corrente do Brasil, e que prevaleceu em grande parte da área estudada com exceção das áreas costeiras. Nesses ambientes dominam organismos nanoplanctônicos adaptados à baixas concentrações de nutrientes devido à sua maior relação superfície: volume, o que lhes possibilita uma alta taxa de crescimento às custas de uma baixa taxa de absorção de nutrientes (EPPLEY et al., 1969; FRIEBELE et al., 1978). Situações semelhantes foram observadas não só no presente trabalho como também em estudos anteriores realizados na mesma região (SOARES, 1983; BRANDINI, 1986). BRANDINI (1986) observou a abundância de cocolitoforídeos nas áreas mais afetadas pela Água Tropical durante o inverno de 1982. As características tropicais da região estudada oferecem condições adequadas de luz, temperatura e pH elevado, necessárias para o desenvolvimento de cocolitoforídeos (PAASCHE, 1968a, 1968b) o que naturalmente explica a grande abundância de Coccolithus huxleyi em quase todas as amostras. 
Ao contrário do que ocorre com células diminutas, as diatomáceas do microplancton necessitam de maiores concentrações de nutrientes para atingir a taxa máxima de crescimento. Apesar de ocorrerem em concentrações comparativamente elevadas nas áreas oceânicas ao norte, estiveram retritas às áreas costeiras da radial de Santa Catarina. SOARES (1983) verificou que Climacodium frauenfeldianum foi a diatomácea dominante na região sueste durante o outono de 1976. Entretanto, no presente trabalho, também realizado no outono, essa espécie foi identificada apenas nas estações 6231 e 6290. De acordo com BRANDINI (1986), os gêneros Chaetoceros e Rhizosolenia foram os que apresentaram um maior número de espécies durante o inverno de 1982. Estes gêneros estiveram bem representados durante o presente trabalho, porém, outros gêneros foram igualmente importantes, tais como Thalassiothrix, Hemialus e Biddulphia.

De acordo com experimentos feitos com culturas de Dictyocha fibula, VAN VALKENBURG \& NORRIS (1970) verificaram que os silicoflagelados estão melhor adaptados à águas menos salinas, mais frias e com concentrações adequadas de silica. Os padrões de distribuição observados vem confirmar estas observações, uma vez que Dictyocha fibula esteve praticamente restrita às águas costeiras do litoral catarinense.

A associação fitoplanctônica da região sueste durante o outono de 1983 é tipicamente indicadora de águas oligotróficas quentes. A predominância de fitoflagelados e cocolitoforídeos nanoplanctônicos, a abundância de cianofíceas filamentosas e a escassez de diatomáceas e dinoflagelados do microplancton, são resultados já anteriormente observados (SOARES, 1983; BRANDINI, 1986) em amostras de regiões afetadas pela massa de Água Tropical da Corrente do Brasil.

\section{AGRADECIMENTOS}

Agradecemos à Diretoria de Hidrografia e Navegação (MM) pela oportunidade de embarque no N/Oc. Almirante Saldanha, e à tripulação do navio pelo auxílio durante as coletas. Ao Dr. Paulo Lana pela leitura e revisão do manuscrito. 


\section{REFERENCIAS}

BRANDINI, F.P. 1982. Variação nictimeral de alguns fatores ecológicos na região de Cananéia (São Paulo). Arq. Biol. Tecnol., 25(3/4). 313-327.

BRANDINI, F.P. 1985. Seasonal succession of the phytoplankton in the Bay of Paranaguá. Rev. Brasil. Biol., 45(4):

BRANDINI, F.P. 1986. Hidrografia e Características do Fitoplâncton da Região Sueste do Brasil: Produção Primária, Biomassa e Composição. Disser. tação de Doutorado, Universidade de São Paulo, Instituto Oceanográfico. $110 \mathrm{p}$.

DHN. 1983. Relatório da OPERAÇÃO SUESTE II. N/Oc. Almirante Saldanha, não publicado.

EDLER, L. 1979. Recommendations on methods for marine biological studies in the Baltic Sea. Phytoplankton and Chlorophyll. The Baltic Marine Biologists Publ. N. 5, 39 p.

EMILSSON, I. 1961. The shelf and coastal waters of southern Brasil. Bolm Inst. oceanogr., S. Paulo, 11(2):101-112.

EPPLEY, R.W.; ROGERS, J.N. \& MCCARTHY, J.J. 1969. Half-saturation constants for uptake of nitrate ammonium by marine phytoplankton. Limnol. Oceanogr. 14:912-920.

FRIEBELE, E.S.; CORFELL, D.L. \& FAUST, M.A. 1978. Relationship between phytoplankton cell size and the rate of orthophosphate uptake: in situ observations of an estuarine population. Mar. Biol., 52:113-122.

KUTNER, M.B. 1974. Seasonal variation and phytoplankton distribution in Cananeia region, Brazil. Publ. N. 361 Inst. Oceanogr. Univ. S. Paulo 17 p.

MESQUITA, A.R.; LEITE, J.B.A. \& RIZZO, R. 1983. Note on the shelf break upwelling off the southeast coast of Brazil (Lat. 26³0'S). Bolm Inst. oceanogr., S. Paulo, 32:193-198.

MIRANDA, L.B.; LUEDMANN, E.F. \& MIYAO, S.Y. 1973. Relatório sobre a segunda pesquisa oceanográfica e pesqueira do Atlântico Sul entre Torres e Maldonado (Lat. $29-35^{\circ} \mathrm{S}$ ). Programa Rio Grande do Sul. II. Distribuição da temperatura, salinidade e circulação geral em superfície. Publ. Esp. Inst. Oceanogr., S. Paulo, 3(II):1-81.

MOREIRA, H.; MOREIRA, I.M.V. \& CECY, I.I.T. 1975. Diatomáceas da Baía de Pananaguá, Paraná, Brasil. Bolm Mus. Bot. Munic. Curitiba $N^{\circ}$ 20, 20p.

MOREIRA, I.M.V.; MOREIRA, H. 1978. Diatomáceas litorais e planctônicas de dezessete estações localizadas entre Ubatuba e Florianópolis, Estado de Santa Catarina, Brasil. Acta Biol. Paranaense, Curitiba, $7(1,2,3,4)$.

PAASCHE, E. 1968a. Biology and physiology of coccolithophorids. Ann. Rev. Microbiol., 22:71-86.

PAASCHE, E. 1968b. The effect of temperature, light intensity and photoper iod on coccolith formation. Limnol. Oceanogr., 13:178-181.

PALACIO, F.J. 1982. Revision zoogeografica marina del sur de Brasil. Bolm Inst. oceanogr., S. Paulo, 31(1):66-92. 
SCOR-UNESCO W.G. 17. 1966. Determination of photosynthetic pigments. Unesco Monogr. Oceanogr. Methodol., 1:9-18.

STRICKLAND, J.D.H. \& PARSONS, T.R. 1972. A Practical Handbook of Seawater Analysis, 2nd ed., Bull. Fish. Res. Bd. Canadá N.॰ 122, 172 p.

UTERMÖHL, H. 1958. Zur Vervollkommnung der quantitativen PhytoplanktonMethodik. Mitt. int. Ver. theor. angew. Limnol., 9:1-38.

VAN VALKENBURG, S.D. \& NORRIS, R.E. 1970. The growth and morphology of the silicoflagellate Dictyocha fibula Ehrenberg in culture. J. Phycol., 6:48-54.

VENRICK, E.L. 1978. How many cells to count? In: Phytoplankton Manual. A. SOURNIA (ed.), UNESCO, 337 p. 


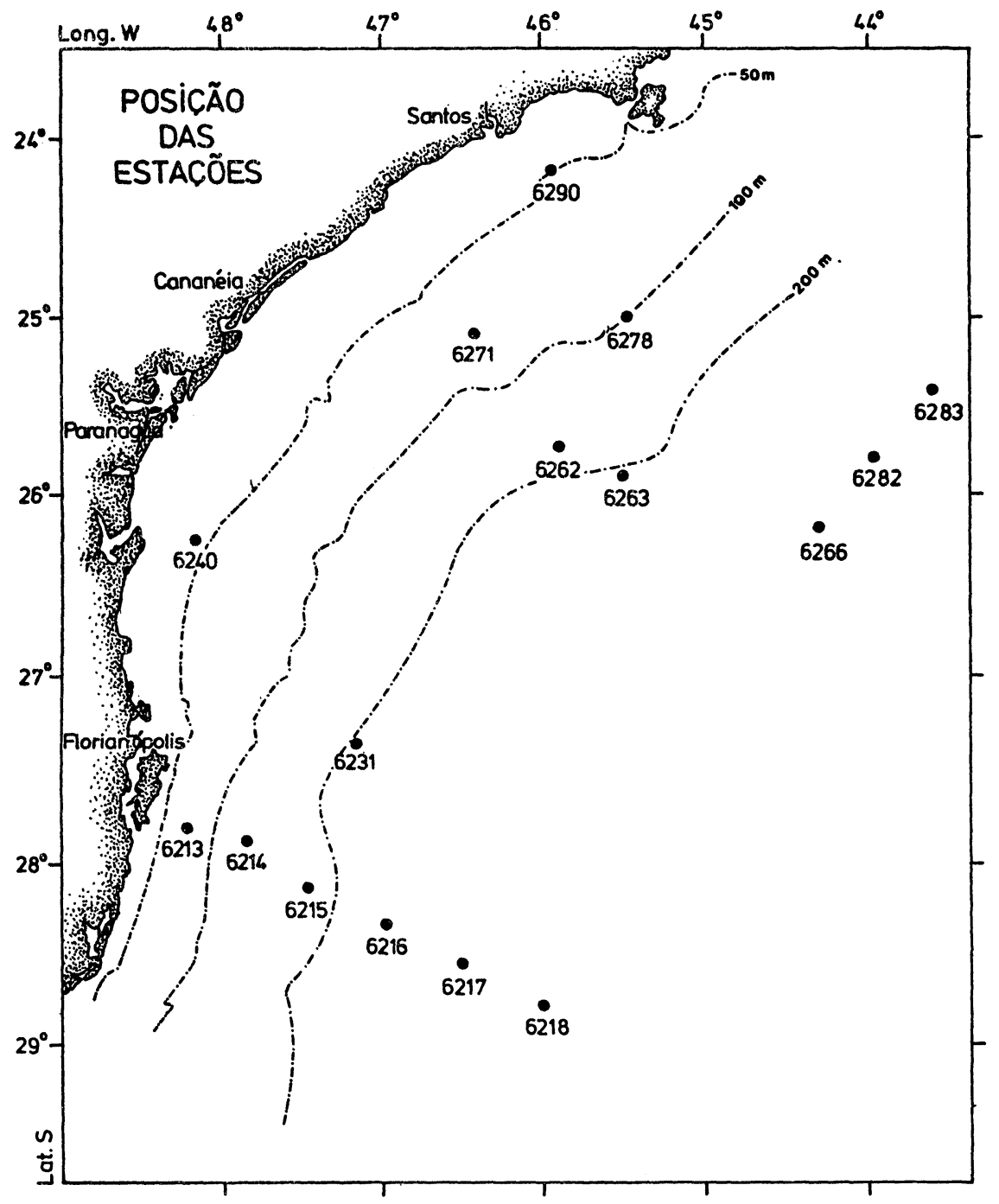

Fig. 1. Mapa da região estudada e posição das estações. 


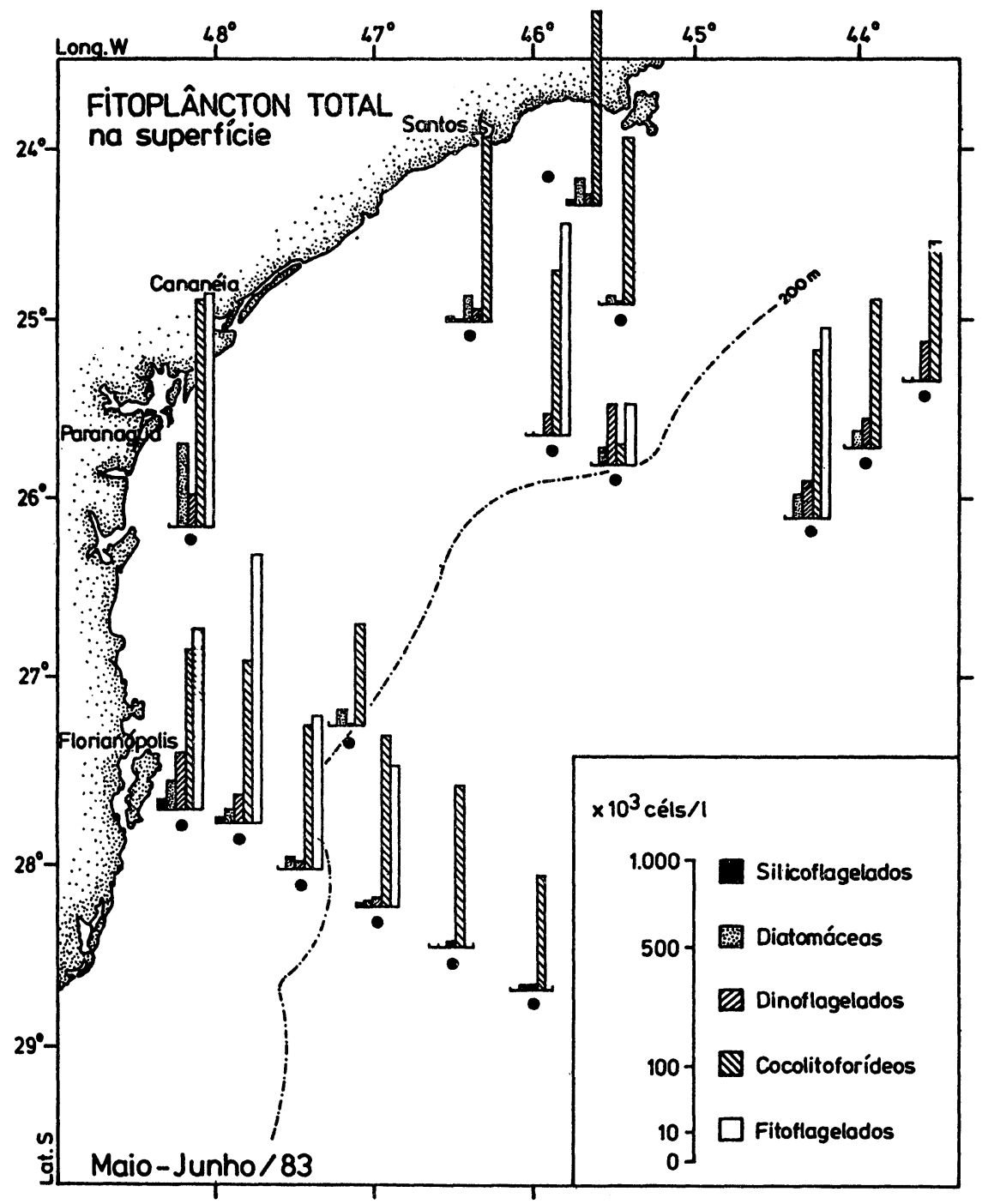

Fig. 2. Concentração e distribuição geográfica dos grupos fitoplanctônicos durante o outono de 1983, na superfície da região sueste. 